Pacific

Journal of

Mathematics

ON THE SIGNATURE OF CERTAIN SPHERICAL REPRESENTATIONS

Carina Boyallian 


\title{
ON THE SIGNATURE OF CERTAIN SPHERICAL REPRESENTATIONS
}

\author{
CARINA BOYALLiAN
}

\begin{abstract}
In this work we prove for all simple real groups of classical type, except for $S O^{*}(n)$ and $S p(p, q)$, that if $\nu \in \mathfrak{a}^{*}$ does not belong to $C_{\rho}$, the convex hull of the Weyl group orbit of $\rho$, then the signature of the Hermitian form attached to the irreducible subquotient of the principal spherical series corresponding to $\nu$, with integral infinitesimal character, is indefinite on $K$-types in $\mathfrak{p}$.
\end{abstract}

\section{Introduction.}

Let $G$ be a real semisimple Lie group. Let $\mathfrak{g}_{0}$ be the Lie algebra of $G$. We will denote the complexification of any vector space $V_{0}$ by $V$ and its dual by $V^{*}$. Let $\mathfrak{g}_{0}=\mathfrak{k}_{0}+\mathfrak{p}_{0}$ be a fixed Cartan decomposition corresponding to the Cartan involution $\theta$ of $\mathfrak{g}_{0}$. Let $K$ be the corresponding maximal compact subgroup of $G$. Let us fix $T \subset K$ a maximal torus. Define $S \subset \mathfrak{t}^{*}$ as the set of weights of $T$ that are sums of distinct non-compact roots.

We say that $\left(\mu, V_{\mu}\right)$ an irreducible representation of $K$ is unitarily small if the weights of $\mu$ lie in the convex hull of $S$. Let us state the following:

Salamanca-Vogan Conjecture. Suppose $X$ is an irreducible Hermitian $(\mathfrak{g}, K)$-module containing a $K$ type in $S$. Then:

(1) If $X$ is unitary, then the real part of the infinitesimal character belongs to the convex hull of the Weyl group orbit $W \cdot \rho$, where $\rho$ is the semi-sum of positive roots.

(2) If $X$ is not unitarizable, then the invariant hermitian form must be indefinite on unitarily small $K$-types.

Using this conjecture the classification of unitary representation can be reduced to the unitarily small case.

If $X$ is a spherical representation, the statement (1) is true by [HJ]. In order to move towards (2) we may assume that real part of the infinitesimal character does not belongs to $W \cdot \rho$, and the hope is that if this holds, the invariant hermitian form is negative definite in the $K$-types in $\mathfrak{p}$. In this work we prove for all simple real groups of classical type, except for $S O^{*}(n)$ and $S p(p, q)$, that if $\nu \in \mathfrak{a}^{*}$ does not belong to $C_{\rho}$, then the signature of the Hermitian form attached to the irreducible subquotient of the principal 
spherical series corresponding to $\nu$, with integral infinitesimal character, is indefinite on $K$-types in $\mathfrak{p}$.

Acknowledgment. I am very grateful to David Vogan, for introducing me in this problem and for fruitful discussions. Special thanks to MIT for the hospitality during my stay there.

\section{Notation and General Results.}

Recall that the complexification of a Lie algebra $\mathfrak{g}_{0}$ will be denoted by $\mathfrak{g}$. Let $\mathfrak{a}_{0} \subset \mathfrak{p}_{0}$ be a maximal Abelian subspace. Let $\Sigma\left(\mathfrak{g}_{0}, \mathfrak{a}_{0}\right)=\Sigma$ be the corresponding set of restricted roots and let $\bar{\Sigma}\left(\mathfrak{g}_{0}, \mathfrak{a}_{0}\right)=\bar{\Sigma}$ be the reduced restricted roots. If $\alpha \in \mathfrak{a}^{*}$, then we will denote the corresponding weight space in $\mathfrak{g}_{0}$ by $\mathfrak{g}_{0}^{\alpha}$ and let $m_{\alpha}$ the dimension of this subspace. For a choice of positive roots $\Sigma^{+}\left(\mathfrak{g}_{0}, \mathfrak{a}_{0}\right)=\Sigma^{+}$we have $\Pi\left(\mathfrak{g}_{0}, \mathfrak{a}_{0}\right)=\Pi$ the set of simple roots. Let $W \simeq N_{K}\left(\mathfrak{a}_{0}\right) \backslash Z_{K}\left(\mathfrak{a}_{0}\right)$ be the corresponding Weyl group. If $w \in W$, then we define $\Sigma^{+}(w)=\left\{\alpha \in \Sigma^{+}: w \alpha \notin \Sigma^{+}\right\}$. We also denote the longest element in the Weyl group by $w_{0}$. If $\Omega \subseteq \Sigma^{+}$then we will say that $\nu \in \mathfrak{a}^{*}$ is it positive (resp. negative) with respect to $\Omega$, if $\operatorname{Re}\langle\alpha, \nu\rangle$ is positive (resp. negative) or zero for all $\alpha \in \Omega$.

If $X$ is an admissible representation of $G$, we will also denote the corresponding $(\mathfrak{g}, K)$-module by X.

Recall that a $(\mathfrak{g}, K)$-module $\left(\pi, H_{\pi}\right)$ is called spherical if the trivial $K$ type occurs in the restriction of $\left(\pi, H_{\pi}\right)$ to $K$, i.e., $H_{\pi}$ contains a non-trivial $K$-fixed vector. Then, we have the following:

Theorem 1.1. The irreducible spherical $(\mathfrak{g}, K)$-modules $\left(\pi, H_{\pi}\right)$ are in one-to-one correspondence with the $W$-orbits in $\mathfrak{a}^{*}$.

A proof of this Theorem appears in [K, BJ2].

This correspondence can be realized as follows: Set $\mathfrak{n}_{0}=\sum_{\alpha \in \Sigma^{+}} \mathfrak{g}_{0}^{\alpha}$ and $\rho=\frac{1}{2} \sum_{\alpha \in \Sigma^{+}} \alpha$. Let $\mathrm{P}=\mathrm{MAN}$ the corresponding minimal parabolic subgroup of $G$. If $\nu \in \mathfrak{a}^{*}$, define

$$
I_{P}^{G}(\nu)=\operatorname{Ind}_{M A N}^{G}(1 \otimes \nu \otimes 1)
$$

where the right-hand-side is the space

$$
\begin{aligned}
& \left\{f \in C^{\infty}(G, \mathbb{C}): \forall \text { man } \in M A N\right. \text { s.t. } \\
& \left.\qquad f(\text { gman })=a^{-\nu-\rho} f(g), f \text { is } K-\text { finite }\right\}
\end{aligned}
$$

and the $\mathfrak{g}$ - action on this induced module is the left regular action, i.e.,:

$$
(X \cdot f)(g)=\frac{d}{d t} f(\exp (-t \cdot X) g)_{\mid t=0}, \quad g \in G, X \in \mathfrak{g}, f \in I_{p}^{G}(\nu)
$$

and

$$
(k \cdot f)(g)=f\left(k^{-1} g\right), \quad k \in K \text { and } f \in I_{P}^{G}(\nu)
$$


It is easy to see that $I_{P}^{G}(\nu)$ is a Harish-Chandra module of finite length. Observe that by the Iwasawa decomposition $G=K A N$ of $G$ we have

$$
I_{P}^{G}(\nu)_{\left.\right|_{K}}=K \text { - finite part of } \operatorname{Ind}_{M}^{K}(1)
$$

and hence by Frobenius reciprocity

$$
\operatorname{dim} I_{P}^{G}(\nu)^{K}=\operatorname{dim} \operatorname{Ind}_{M}^{K}(1)^{K}=\operatorname{dim} \operatorname{Hom}(\mathbb{C}, \mathbb{C})=1 .
$$

So $I_{P}^{G}(\nu)$ is a spherical $(\mathfrak{g}, K)$-module, and by (1.1) there exists a unique irreducible composition factor $J^{G}(\nu)=J_{P}^{G}(\nu)$ containing the trivial $K$-type in $I_{P}^{G}(\nu)$. It is well-known that $J^{G}(\nu) \simeq J^{G}\left(\nu^{\prime}\right)$ if and only if there exists $w \in W$ such that $\nu=w \cdot \nu^{\prime}$, in particular $J^{G}(\nu)$ does not depend on the choice of the minimal parabolic subgroup containing $M A$. The $(\mathfrak{g}, K)$ modules $I_{P}^{G}(\nu)$ are called spherical principal series representations.

We denote the set of $\nu \in \mathfrak{a}^{*}$ such that $J^{G}(\nu)$ has integral infinitesimal character by $\mathfrak{a}_{\text {int }}^{*}$.

Let $X$ be a $(\mathfrak{g}, K)$-module. Then we say that $X$ admits an invariant Hermitian form if there exists a non-zero map $\omega=\omega_{G}: X \times X \longrightarrow \mathbb{C}$ such that:

(1) $\omega$ is linear in the first factor and conjugate linear in the second factor.

(2) $\omega(x, y)=\overline{\omega(y, x)}, \quad x, y \in X$.

(3) $\omega(k \cdot x, k \cdot y)=\omega(x, y), \quad x, y \in X, \quad k \in K$.

(4) $\omega(H \cdot x, y)=\omega(x,-\bar{H} \cdot y), \quad x, y \in X, \quad H \in \mathfrak{g}$, where $\bar{H}$ stand for complex conjugation of $\mathfrak{g}$ respect to $\mathfrak{g}_{0}$.

If $\mu, \mu^{\prime}$ are two different $K$-types, then, (3) implies that $\omega\left(X^{\mu}, X^{\mu^{\prime}}\right)=0$. Hence $\omega$ is completely described by its restriction to the $K$-isotopic spaces on $X$.

Let $\mu \in \hat{K}$. Then $X^{\mu} \simeq \operatorname{Hom}_{K}\left(V_{\mu}, X\right) \otimes V_{\mu}$ and $\omega$ induces a Hermitian form, $\omega^{\mu}$, on the first factor.

Definition 1.3. Let $(p(\mu), q(\mu)):=\left(p_{X}(\mu), q_{X}(\mu)\right):=\left(p_{X}^{G}(\mu), q_{X}^{G}(\mu)\right)$ be the signature of $\omega^{\mu}$, i.e., $p(\mu)$ (respective $q(\mu)$ ) is the sum of the strictly positive (respective negative) eigenspaces of $\omega^{\mu}$.

Let us see when there exists this Hermitian form in $J(\nu)$. This is the one being used in this article. Choose a minimal parabolic subgroup $P=M A N$ in $G$. Consider $w \in W$ and $\nu$ positive with respect to $\Sigma^{+}(w)$. Then there exist an intertwining operator $\Psi(w): \mathrm{I}_{P}^{G}(\nu) \longrightarrow \mathrm{I}_{P}^{G}(w \nu)$ so that $\Psi(w)$ is an isomorphism on the trivial $K$-type. If $w=w_{0}$ then the image is isomorphic to $J(\nu)$.

Recall that there is a natural non-zero Hermitian paring

$$
\mathrm{I}_{P}^{G}(\nu) \times \mathrm{I}_{P}^{G}(-\bar{\nu}) \stackrel{\langle,\rangle}{\longrightarrow} \mathbb{C} .
$$


Here $\mathrm{I}_{M A N}^{G}(\nu)$ is naturally identified with the $K$-finite part of $L^{2}(K / M)$ and with this identification, $\langle$,$\rangle is the inner product on L^{2}(K / M)$. Now suppose there exists $w \in W$ such that $-\bar{\nu}=w \nu$ and $\nu$ is positive with respect to $M A N$. Then we get the Hermitian pairing

$$
\begin{aligned}
\mathrm{I}_{P}^{G}(\nu) \times \mathrm{I}_{P}^{G}(\nu) & \stackrel{\omega_{\nu}}{\longrightarrow} \mathbb{C} \\
\left(v_{1}, v_{2}\right) & \longrightarrow\left\langle v_{1}, \Psi(w) v_{2}\right\rangle .
\end{aligned}
$$

Since $\Psi\left(w \nu, w_{0} w^{-1}\right): \mathrm{I}_{P}^{G}(w \nu) \longrightarrow \mathrm{I}_{P}^{G}\left(w_{0} \nu\right)$ is an isomorphism, we get that $\mathrm{I}_{M A N}^{G}(\nu) / \operatorname{Rad} \omega \simeq J^{G}(\nu)$. Hence $\omega$ induces a Hermitian form on $J(\nu)$. The same argument shows that $J(\nu)$ admits an invariant Hermitian form if and only if $-\bar{\nu}$ is $W$-conjugate to $\nu$.

Now, we will define the set of K-types where we will work. So, let's define as the $\mathfrak{p}_{0}$-representation of $K$ the homomorphism

$$
\begin{aligned}
& K \longrightarrow G L\left(\mathfrak{p}_{0}\right) \\
& k \longmapsto A d(k)_{\left.\right|_{\mathfrak{p}_{0}}},
\end{aligned}
$$

and the $\mathfrak{p}$-representation of $K$ the complexification of $\mathfrak{p}_{0}$-representation.

Recall that if $\mathfrak{g}_{0}$ is simple, then $\mathfrak{p}$ is either irreducible or it is a direct sum of two inequivalent irreducible representations. Consider the following set of $K$-types:

$$
\mathfrak{p}=\left\{\mu \in \hat{K}: \operatorname{Hom}_{K}(\mu, \mathfrak{p}) \neq 0\right\} .
$$

Through this work, we will consider the signature over this set of $K$-types.

Finally we will define $C_{\rho}$ as the convex hull of points $w \rho$ with $w \in W$. This set is also characterized in terms of positive roots by the following:

Proposition 1.5. The set $C_{\rho}$ coincides with the set of all weights of the form

$$
r=\sum_{\alpha \in \Sigma^{+}} c_{\alpha} \alpha \quad \text { where }-1 / 2 \leq c_{\alpha} \leq 1 / 2,
$$

and each $\alpha$ is counted with multiplicity.

For a proof of this result we refer to $[\mathbf{S V}]$.

\section{Problem.}

Take $\nu \in \mathfrak{a}_{i n t}^{*}$ such that $J(\nu)$ has integral infinitesimal character and admits an invariant Hermitian form. Then, we will prove the following

Theorem 2.1. If $\nu \notin C_{\rho}$ then $q_{\mu}(\nu)>0$, where $\mu$ is a $K$-type in $\mathfrak{p}$ and $q$ as in Definition 1.3. 
The main problem here is that this Hermitian form has no known general expression. However, Bang-Jensen [BJ], proves some useful Theorems, that give conditions for an invariant Hermitian form on an irreducible spherical representation, with integral infinitesimal character, to be positive definite on the $K$-types $\mu \in \mathfrak{p}$, in terms of Langlands data, for the simple groups of classical type except $S O^{*}(n)$ and $S p(p, q)$.

These Theorems will be the main tool we will use in order to prove Theorem 2.1. This will be done case by case. Bang-Jensen's results are used to get an explicit characterization of $\nu \in \mathfrak{a}^{*}$, that is crucial for the proof of Theorem 2.1.

\section{Case $S L(n, \mathbb{F})$ with $\mathbb{F}=\mathbb{R}$ or $\mathbb{C}$.}

Let $\mu$ be a $K$-type in $\mathfrak{p} \cap[\mathfrak{g}, \mathfrak{g}]$. We identify $\mathfrak{a}^{*}$ with $\mathbb{C}^{n}$ such that $\Sigma\left(\mathfrak{g}_{0}, \mathfrak{a}_{0}\right)=$ $\left\{ \pm\left(e_{i}-e_{j}\right) \mid 1 \leq i<j \leq n\right\}$. Then $\Sigma^{+}\left(\mathfrak{g}_{0}, \mathfrak{a}_{0}\right)=\left\{\left(e_{i}-e_{j}\right) \mid 1 \leq i<j \leq n\right\}$. Put $r=\operatorname{dim}_{\mathbb{R}} \mathbb{F}$. Then, $\operatorname{dim} \mathfrak{g}_{0}^{\alpha}=r$. Then $\mathfrak{a}_{\text {int }}^{*}=\left\{\nu \in \mathbb{C}^{n} \mid \nu_{i} \equiv 0\right.$ $\bmod r \mathbb{Z}, i=1, \ldots, n\}$. For $\nu \in \mathfrak{a}_{\text {int }}^{*}$ and $i \in \mathbb{Z}$ we define

$$
R(i):=R_{\nu}(i):=\#\left\{\nu_{j}: \nu_{j}=r \cdot i\right\} .
$$

Now if $\nu \in \mathfrak{a}_{\text {int }}^{*}$, then $J^{G}(\nu)$ admits an invariant Hermitian form if and only if $R_{\nu}(i)=R_{\nu}(-i)$ for all $i$.

Then we have the following:

Theorem 3.1. Assume $\mathbb{F}=\mathbb{C}$ or $\mathbb{R}$. Suppose $\nu \in \mathfrak{a}_{\mathrm{int}}^{*}$ and $J^{G}(\nu)$ admits an invariant hermitian form, $\omega$. Then $\omega$ is positive definite on $J^{G}(\nu)^{\mu}$ if and only if $R_{\nu}(i+1) \leq R_{\nu}(i)$ for all $i \geq 0$.

Proof. See Theorem 5.2 in $[\mathbf{B J}]$.

We will now prove the following proposition.

Proposition 3.2. Consider $\nu \in \mathfrak{a}_{\mathrm{int}}^{*}$ such that $J(\nu)$ has integral infinitesimal character. If $R_{\nu}(i+1) \leq R_{\nu}(i)$ for all $i \geq 0$ then $\nu=\sum_{\alpha \in \Sigma^{+}} c_{\alpha} \alpha$ with $c_{\alpha}=1 / 2$ or 0 .

Proof. We will give the proof only for $\operatorname{Sl}(n, \mathbb{R})$. The case $\operatorname{Sl}(n, \mathbb{C})$ follows immediately from the definition of $\mathfrak{a}_{\mathrm{int}}^{*}$ and the fact that for this group, each root has multiplicity 2 . Take $\nu \in \mathfrak{a}_{\text {int }}^{*}$ such that:

(1) $R_{\nu}(i+1) \leq R_{\nu}(i)$ for all $i \geq 0$,

(2) $R_{\nu}(i)=R_{\nu}(-i)$ for all $i$;

thus, we are assuming that $\omega$ is positive definite on $J^{G}(\nu)^{\mu}$. By definition, $C_{\rho}$ is stable by Weyl group action, hence by (1) and (2) we can consider,

$$
\begin{array}{r}
\nu=(k, \ldots, k, k-1, \cdots, k-1, \cdots \cdots, 1, \cdots, 1,0, \cdots, 0,-1, \cdots,-1, \cdots \\
\ldots \cdots,-(k-1), \cdots,-(k-1),-k, \cdots,-k)
\end{array}
$$


where $k \leq\left[\frac{n}{2}\right]-1$.

In order to complete this proof, we will need the following:

Lemma 3.4. Take $\nu$ as above and consider $R(0) \geq 1$ (otherwise, $\nu \equiv 0$ by (1)). Then

$$
2(k-i)-2<n-\left(2 \sum_{j=0}^{i} R(k-j)\right)+1 \quad \text { where } i=0, \ldots, k-1
$$

Proof. Since $n=2 \sum_{j=0}^{k-1} R(k-j)+R(0)$ we have

$$
\begin{aligned}
2(k-i)-2 \leq 2 \sum_{j=i+1}^{k-1} R(k-j)+R(0) & =n-2 \sum_{j=0}^{i} R(k-j) \\
& <n-2 \sum_{j=0}^{i} R(k-j)+1 .
\end{aligned}
$$

Then, (3.5) has been proved.

Proof of Proposition 3.2 (Continuation). Let define

$$
\mathcal{T}(m)=\left\{\begin{array}{cl}
\sum_{t=0}^{m-1} R(k-t), & \text { if } m>0 \\
0, & \text { if } m=0
\end{array} .\right.
$$

Now, it is easy to check, that Lemma 3.4 allows us to rewrite $\nu$ as follows:

$$
\begin{aligned}
\nu=\frac{1}{2} & \sum_{m=0}^{k-1} \sum_{j=1}^{R(k-m)}\left\{\left(e_{\mathcal{T}(m)+j}-e_{n-\mathcal{T}(m)-j+1}\right)\right. \\
& \left.+\sum_{s=\mathcal{T}(m)+j}^{2(k-m)-2+\mathcal{T}(m)+j}\left[\left(e_{\mathcal{T}(m)+j}-e_{s+1}\right)+\left(e_{s+1}-e_{n-\mathcal{T}(m)-j+1}\right)\right]\right\} .
\end{aligned}
$$

And so, Proposition 3.2 is proved.

Proof of Theorem 2.1 for $\operatorname{Sl}(n, \mathbb{F})$ with $\mathbb{F}=\mathbb{R}, \mathbb{C}$. This is immediate from Proposition 3.2, Theorem 3.1 and Proposition 1.5.

\section{Case $S p(2 n, \mathbb{F}), n>2$ with $\mathbb{F}=\mathbb{R}, \mathbb{C}$.}

Now, assume that $G=S p(2 n, \mathbb{R})$ or $G=S p(2 n, \mathbb{C})$. In this case, $\Sigma\left(\mathfrak{g}_{0}, \mathfrak{a}_{0}\right)$ is of type $C_{n}$, and identifying $\mathfrak{a} \simeq \mathbb{C}^{n}$ we have that the restricted roots are $\left\{ \pm e_{i} \pm e_{j}, \pm 2 e_{l}\right\}$. Put $r=\operatorname{dim}_{\mathbb{R}} \mathbb{F}$.

Here, $J^{G}(\nu)$ has integral infinitesimal character if and only if $\nu_{i} \in r \mathbb{Z}$. Take $\nu \in \mathfrak{a}^{*}$, and replace it by a Weyl group conjugate, then we may assume 
that

$$
\nu_{1} \geq \nu_{2} \geq \cdots \geq \nu_{n} \geq 0
$$

and with this assumption define

$$
R(i)=R_{\nu}(i)=\#\left\{j: \nu_{j}=r i\right\}, \quad i \geq 0 .
$$

Then we can state the following:

Theorem 4.2. If $J(\nu)$ has integral infinitesimal character, then an invariant Hermitian form is positive definite on $J(\nu)^{\mu}, \mu \in \mathfrak{p}$, if and only if the following conditions are satisfied:

(1) $R(i+1) \leq R(i)+1$, for $i \geq 1$;

(2) If $R(i+1)=R(i)+1$, for $i \geq 1$, then $R(i)$ is odd;

(3) $R(1) \leq 2 R(0)+2$.

Proof. See Theorem 8.4 in $[\mathbf{B J}]$.

Now, we can prove the following:

Proposition 4.3. Consider $\nu \in \mathfrak{a}_{\mathrm{int}}^{*}$ such that $J(\nu)$ has integral infinitesimal character. If conditions (1)-(3) above are satisfied then $\nu=\sum_{\alpha \in \Sigma^{+}} c_{\alpha} \alpha$ with $c_{\alpha}=1 / 2$ or 0 .

Proof. Let us assume that $G=S p(2 n, \mathbb{R})$ and so $r=1$. It follows by Condition (2) in Theorem 4.2, that if $R(j)=0$ for any $j \geq 1$ then $R(j+1)=$ 0 . This implies that if $R(1) \neq 0$ then

$$
\nu=(k, \ldots, k, k-1, \ldots, k-1, \ldots, \ldots, 1, \ldots, 1,0, \ldots, 0)
$$

or

$$
\nu=(k, \ldots, k, k-1, \ldots, k-1, \ldots, \ldots, 1)
$$

where $k \leq n$. Now, since $n=\sum_{j=0}^{k} R(k-j)$, we have that

$$
k-j \leq n-\sum_{i=0}^{j} R(k-i)
$$

with $j=0, \ldots, k-2$. Let us define $\mathcal{T}(m)$ as in (3.6), and Inequality 4.4 allows us to write $\nu$ as follows

$$
\begin{aligned}
\nu= & \frac{1}{2} \sum_{m=0}^{k-2} \sum_{j=1}^{R(k-m)}\left\{2 e_{\mathcal{T}(m)+j}\right. \\
& \left.+\sum_{s=\mathcal{T}(m)+j+1}^{(k-m)-1+\mathcal{T}(m)+j}\left[\left(e_{\mathcal{T}(m)+j}-e_{s}\right)+\left(e_{\mathcal{T}(m)+j}+e_{s}\right)\right]\right\} \\
& +\frac{1}{2} \sum_{j=1}^{R(1)} 2 e_{\mathcal{T}(k-1)+j .}
\end{aligned}
$$


The case $G=S p(2 n, \mathbb{C})$ follows from this using that $r=2$ and the multipilcities of the positive roots. Hence, in this way we have proved Proposition 4.3 .

Now, we can give the:

Proof of Theorem 2.1 for $S p(2 n, \mathbb{F})$ with $\mathbb{F}=\mathbb{R}, \mathbb{C}$. It follows from Proposition 4.3, Theorem 4.2 and Proposition 1.5.

5. Case $S O(p, q), p>q+1$ and $S U(p, q), p>q$.

Let us assume that $G=S O(p, q), p>q+1$ or $G=S U(p, q), p>q$; and as usual identify $\mathfrak{a} \simeq \mathbb{C}^{q}$. Then the restricted roots become $\left\{ \pm e_{i} \pm e_{j}, \pm e_{l}\right\}$. Define

$$
r= \begin{cases}1, & \text { if } G=S O(p, q) \\ 2, & \text { if } G=S U(p, q) .\end{cases}
$$

Define, $\epsilon=0,1$ by $\epsilon \equiv p-q-1+r \bmod 2 \mathbb{Z}$. In these cases, $J(\nu)$ has integral infinitesimal character if and only if

$$
2 \nu_{i} \equiv r \epsilon \bmod 2 r \mathbb{Z}, \quad i=1, \ldots, q .
$$

We can replace $\nu$ by a Weyl group conjugate, and assume that

$$
\nu_{1} \geq \nu_{2} \geq \cdots \geq \nu_{n} \geq 0 \text {. }
$$

With this assumption we define

$$
R(i)=R_{\nu}(i)=\#\left\{j: \nu_{j}=r\left(i+\frac{\epsilon}{2}\right)\right\}, \quad i \geq 1
$$

and

$$
R(0)=R_{\nu}(0)=(2-\epsilon) \#\left\{j: \nu_{j}=r\left(\frac{\epsilon}{2}\right)\right\} .
$$

Take $s=\frac{p-q-1+r-\epsilon}{2}$. Now, we can state the following:

Theorem 5.4. If $J(\nu)$ has integral infinitesimal character, then an invariant Hermitian form is positive definite on $J(\nu)^{\mu}, \mu \in \mathfrak{p}$, if and only if the following conditions are satisfied:

(1) $R(i+1) \leq R(i)+1$, for $i \geq 0, i \neq s-1$;

$R(i+1)=R(i)+1$, for $i \geq 0, i \neq s-1$ then $\left\{\begin{array}{l}R(i) \text { is even, } \quad \text { if } i<s \\ R(i) \text { is odd, } \quad \text { if } i>s ;\end{array}\right.$

(3) $R(s) \leq R(s-1)+2$;

(4) $R(s)=R(s-1)+2$, then $R(s-1)$ is even.

Proof. Cf. Theorem 6.2 in [BJ].

Now, we can prove the following: 
Proposition 5.5. Consider $\nu \in \mathfrak{a}_{\text {int }}^{*}$ such that $J(\nu)$ has integral infinitesimal character. If conditions (1)-(4) above are satisfied then $\nu=\sum_{\alpha \in \Sigma^{+}} c_{\alpha} \alpha$ with $c_{\alpha}=1 / 2$ or 0 .

Proof. Assume that $r=1$ and $\epsilon=0$, in other words that $G=S O(p, q)$ with $p-q$ even. So $s=\frac{p-q}{2} \in \mathbb{Z}$. In this setting $J(\nu)$ has integral infinitesimal character if and only if $\nu_{i} \in \mathbb{Z}$ and

$$
R(i)=\#\left\{n: \nu_{n}=i\right\} \quad i \geq 1
$$

and

$$
R(0)=2 \#\left\{n: \nu_{n}=0\right\} .
$$

Then by conditions (1)-(4), Formula (5.1) and assuming $R(s) \neq 0$ and there exists $j<s$ such that $R(j) \neq 0$ we can consider $\nu$ as follows

$$
\begin{array}{r}
\nu=(s+k, \ldots, s+k, s+(k-1), \ldots, s+(k-1), \ldots \ldots, s, \ldots, s, \\
\left.\nu_{j}, \ldots, \nu_{j}, \ldots, \nu_{1}, \ldots \nu_{1}\right)
\end{array}
$$

with $0 \leq \nu_{i}<s, i=1, \ldots, j$.

In order to complete the proof of this proposition, we will need the following:

Lemma 5.7. Take $\nu$ as above. Then

$$
k-i+1 \leq q-\sum_{r=0}^{i} R(s+k-r) .
$$

Proof. Since $R(s) \neq 0$ we have, by condition (2) in Theorem $5.4 R(s+m) \neq$ 0 , for $m=1, \ldots, k$. Hence, using that $q=\sum_{r=0}^{k} R(s+k-r)+\sum_{p=0}^{j-1} R\left(\nu_{j-p}\right)$ we have

$$
\begin{aligned}
k-i+1 & \leq \sum_{r=i+1}^{k} R(s+k-r)+\sum_{p=0}^{j-1} R\left(\nu_{j-p}\right) \\
& =q-\sum_{r=0}^{i} R(s+k-r) .
\end{aligned}
$$

So, the proof of the lemma is complete.

Proof Proposition 5.5. (Cont.) Recalling that each root $e_{i} \pm e_{j}$ has multiplicity 1 and $e_{l}$ has multiplicity $p-q=2 s$, and defining

$$
\mathcal{T}(m)=\left\{\begin{array}{cc}
\sum_{i=0}^{m-1} R(s+k-i), & \text { if } m \geq 1 \\
0, & \text { if } m=0
\end{array}\right.
$$

and

$$
\mathcal{S}(n)=\left\{\begin{array}{cc}
\sum_{i=0}^{n-1} R\left(\nu_{j-i}\right), & \text { if } n \geq 1 \\
0, & \text { if } n=0
\end{array}\right.
$$


we can, since is easy to check that $k-m \leq q-\sum_{j=0}^{m} R(s+k-j)$ for $m=0, \ldots, k-1$ and $\nu_{i}<s$ for $i=1, \ldots, j$, rewrite $\nu$ as follows

$$
\begin{aligned}
& \nu=\left(\sum _ { m = 0 } ^ { k - 1 } \sum _ { r = 1 } ^ { R ( s + k - m ) } \frac { 1 } { 2 } \left\{\sum _ { t = 1 } ^ { k - m } \left[\left(e_{\mathcal{T}(m)+r}-e_{\mathcal{T}(m)+r+t}\right)\right.\right.\right. \\
& \left.\left.\left.+\left(e_{\mathcal{T}(m)+r}+e_{\mathcal{T}(m)+r+t}\right)\right]\right\}+s e_{\mathcal{T}(m)+r}\right)+\sum_{r=1}^{R(s)} s e_{\mathcal{T}(s-1)+r} \\
& +\sum_{n=0}^{j-1} \sum_{p=1}^{R\left(\nu_{j-n)}\right.} \nu_{j-n} e_{\mathcal{T}(k+1)+\mathcal{S}(n)+p} .
\end{aligned}
$$

Now let us keep $r=1$ and consider $\epsilon=1$, i.e., $G=S O(p, q)$, but now, $p-q$ is odd. Here $s=\frac{p-q-1}{2}$ and $J(\nu)$ has integral infinitesimal character if and only if $\nu_{n}=\frac{2 l_{n}+1}{2}$ with $l_{n} \in \mathbb{Z}_{+}$and $n=1, \ldots, q$. Then, we have

$$
R(i)=\#\left\{n: \nu_{n}=\frac{2 i+1}{2}\right\} \quad i \geq 1
$$

and

$$
R(0)=\#\left\{n: \nu_{n}=\frac{1}{2}\right\}
$$

Again, as before, we can assume that

$$
\begin{aligned}
\nu= & \left(\frac{2(s+k)+1}{2}, \ldots, \frac{2(s+k)+1}{2}, \frac{2(s+(k-1))+1}{2},\right. \\
& \ldots, \frac{2(s+(k-1))+1}{2}, \ldots, \frac{2 s+1}{2}, \ldots, \frac{2 s+1}{2}, \frac{2 l_{j}+1}{2}, \\
& \left.\ldots, \frac{2 l_{j}+1}{2}, \ldots, \frac{2 l_{1}+1}{2}, \ldots, \frac{2 l_{1}+1}{2}\right)
\end{aligned}
$$

with $0 \leq l_{i}<s, i=1, \ldots, j$. By Lemma 5.7 and since $l_{i}+\frac{1}{2}<s$, we can rewrite $\nu$ as follows

$$
\begin{aligned}
\nu= & \left(\sum _ { m = 0 } ^ { k } \sum _ { r = 1 } ^ { R ( s + k - m ) } \frac { 1 } { 2 } \sum _ { t = 0 } ^ { k - m } \left[\left(e_{\mathcal{T}(m)+r}-e_{\mathcal{T}(m)+r+t+1}\right)\right.\right. \\
& \left.\left.+\left(e_{\mathcal{T}(m)+r}+e_{\mathcal{T}(m)+r+t+1}\right)\right]+\left(s-\frac{1}{2}\right) e_{\mathcal{T}(m)+r}\right) \\
& +\sum_{n=0}^{j} \sum_{u=1}^{R\left(l_{j-n}\right)}\left(l_{j-n}+\frac{1}{2}\right) e_{\mathcal{T}(k+1)+\mathcal{S}(n)+u} .
\end{aligned}
$$

When $R(s)=0$, condition (2) in Theorem 5.4 implies that $R(s+j)=0$ for all $j$. Then this case or when $R\left(\nu_{i}\right)=0$ for all $i$, can be easily deduced from the cases above, and hence, we have completed this proof for $S O(p, q)$ 
and $r=1$ The cases corresponding to $S U(p, q)$ follows almost immediately from this cases above using, as before, the multiplicities of the positive roots for this group.

Then we can give the:

Proof of Theorem 2.1 for $S O(p, q), p>q+1$ and $S U(p, q), p>q$. Is immediate from Proposition 5.5, Theorem 5.4 and Proposition 1.5.

$$
\text { 6. Case } S U(n, n), n \geq 2 \text {. }
$$

In this case, identifying $\mathfrak{a} \simeq \mathbb{C}^{n}$ we have that the restricted roots are $\left\{ \pm e_{i} \pm\right.$ $\left.e_{j}, \pm 2 e_{l}\right\} . J^{G}(\nu)$ has integral infinitesimal character if and only if $\nu_{i} \equiv$ $\epsilon \bmod 2 \mathbb{Z}, \epsilon=0,1$. Take $\nu \in \mathfrak{a}^{*}$. Again, we may assume that

$$
\nu_{1} \geq \nu_{2} \geq \cdots \geq \nu_{n} \geq 0 \text {. }
$$

Hence we define

$$
R(i)=R_{\nu}(i)=\#\left\{j: \nu_{j}=2 i+\epsilon\right\}, \quad i \geq 1
$$

and

$$
R(0)=(2 \epsilon) \#\left\{j: \nu_{j}=\epsilon\right\} .
$$

Now, we can state the following:

Theorem 6.4. If $J(\nu)$ has integral infinitesimal character, then an invariant Hermitian form is positive definite on $J(\nu)^{\mu}, \mu \in \mathfrak{p}$, if and only if the following conditions are satisfied:

(1) $R(i+1) \leq R(i)+1$, for $i \geq 0$;

(2) $R(i+1)=R(i)+1$, then $R(i)$ is odd.

Proof. See Theorem 7.1 in $[\mathbf{B J}]$.

With this, we can prove the following:

Proposition 6.5. Consider $\nu \in \mathfrak{a}_{\text {int }}^{*}$ such that $J(\nu)$ has integral infinitesimal character. If conditions (1)-(2) above are satisfied then $\nu=\sum_{\alpha \in \Sigma^{+}} c_{\alpha} \alpha$ with $c_{\alpha}=1 / 2$ or 0 .

Proof. Let us first assume that $\epsilon=0$. By (6.3) we have that $R(0)=0$. Then it follows from condition (2) in Theorem 6.4 that $\nu \equiv 0$. So we can suppose that $\epsilon=1$ and again, by condition (2), we can consider that

$$
\nu=(2 k+1, \ldots, 2 k+1, \ldots \ldots, 1, \ldots, 1)
$$

with $R(0) \geq 2$. Since $n=\sum_{i=0}^{k-1} R(k-i)+\frac{1}{2} R(0)$, we have

$$
k-m<n-\sum_{i=0}^{m} R(k-i), \quad m=0, \ldots, k-1
$$


and this formula plus the fact that each root $e_{i} \pm e_{j}$ has multiplicity 2 and each $2 e_{l}$ has multiplicity one, allows us to say that

$$
\begin{aligned}
& \nu=\left(\sum_{m=0}^{k-1} \sum_{r=1}^{R(k-m)} \sum_{t=1}^{k-m}\left[\left(e_{\mathcal{T}(m)+r}-e_{\mathcal{T}(m)+r+t}\right)+\left(e_{\mathcal{T}(m)+r}+e_{\mathcal{T}(m)+r+t}\right)\right]\right. \\
& \left.\quad+e_{\mathcal{T}(m)+r}\right)+\sum_{r=1}^{\frac{1}{2} R(0)} e_{\mathcal{T}(k)+r}
\end{aligned}
$$

and in this way, we have completed this proposition.

Hence, we can give the:

Proof of Theorem 2.1 for $S U(n, n), n>2$. It is immediate from Proposition 6.5, Theorem 6.4 and Proposition 1.5.

$$
\text { 7. Case } S O(n+1, n) \text { and } S O(2 n+1, \mathbb{C}), n \geq 2 \text {. }
$$

Let us assume that $G=S O(n+1, n)$ or $G=S O(2 n+1, \mathbb{C})$, and as usual identify $\mathfrak{a} \simeq \mathbb{C}^{n}$. Then the restricted roots become $\left\{ \pm e_{i} \pm e_{j}, \pm e_{l}\right\}$. Define

$$
r= \begin{cases}1, & \text { if } G=S O(n+1, n) \\ 2, & \text { if } G=S O(2 n+1, \mathbb{C}) .\end{cases}
$$

In these cases, $J(\nu)$ integral infinitesimal character if and only if

$$
\nu_{i} \in r \mathbb{Z}+r \frac{\epsilon}{2}, \text { with } \epsilon=0,1 .
$$

We can replace $\nu$ by a Weyl group conjugate, and assume that

$$
\nu_{1} \geq \nu_{2} \geq \cdots \geq \nu_{n} \geq 0
$$

and so, we define

$$
R(i)=R_{\nu}(i)=\#\left\{j: \nu_{j}=r\left(i+\frac{\epsilon}{2}\right)\right\}, \quad i \geq 1
$$

and

$$
R(0)=R_{\nu}(0)=(2-\epsilon) \#\left\{j: \nu_{j}=r \frac{\epsilon}{2}\right\} .
$$

Let us see the following:

Theorem 7.4. If $J(\nu)$ has integral infinitesimal character, then an invariant Hermitian form is positive definite on $J(\nu)^{\mu}, \mu \in \mathfrak{p}$, if and only if the following conditions are satisfied:

(1) $R(i+1) \leq R(i)+1$, for $i \geq 0$

(2) if $R(i+1)=R(i)+1$, for $i \geq 1$, then $R(i)$ is odd;

(3) $R(0)>2-\epsilon$. 
Proof. Cf. Theorem 9.4 in $[\mathbf{B J}]$. The condition $R(0)>2-\epsilon$ does not appear in $[\mathbf{B J}]$. However, it is easy to see by inspection of the proof that this condition is needed.

Now, we can prove the following:

Proposition 7.5. Consider $\nu \in \mathfrak{a}_{\text {int }}^{*}$ such that $J(\nu)$ has integral infinitesimal character. If conditions (1)-(3) above are satisfied then $\nu=\sum_{\alpha \in \Sigma^{+}} c_{\alpha} \alpha$ with $c_{\alpha}=1 / 2$ or 0 .

Proof. Let us assume that $\mathrm{r}=1$. The other case, $\mathrm{r}=2$, follows from this one. And also consider $\epsilon=0$, so here we have that $J(\nu)$ admits integral infinitesimal character if and only if $\nu_{i} \in \mathbb{Z}$. Hence by condition (2) in Theorem 7.4 we have that

$$
\nu=(k, \ldots, k, k-1, \ldots, k-1, \ldots \ldots, 1, \ldots, 1,0, \ldots, 0) .
$$

As before, since $R(0) \geq 2$, we can prove that $k-i \leq n-\sum_{j=0}^{i} R(k-j)$, and this inequality allows us to write down

$$
\nu=\frac{1}{2} \sum_{m=0}^{k-1} \sum_{r=1}^{R(k-m)} \sum_{t=1}^{k-m}\left[\left(e_{\mathcal{T}(m)+r}-e_{\mathcal{T}(m)+r+t}\right)+\left(e_{\mathcal{T}(m)+r}+e_{\mathcal{T}(m)+r+t}\right)\right]
$$

where $\mathcal{T}(m)$ is defined in (3.6). The case $\epsilon=1$ follows from this one, using that

$$
\nu=\nu_{1}+\left(\frac{1}{2}, \ldots, \frac{1}{2}\right)
$$

with $\nu_{1}$ as in (7.6). Since we do not use $e_{i}$ 's in the case above, we can put

$$
\nu=\nu_{1}+\frac{1}{2} \sum_{i=1}^{n} e_{i}
$$

and we have completed the proof of this proposition.

Then we have:

Proof of Theorem 2.1 for $S O(n+1, n)$ and $S O(2 n+1, \mathbb{C}), n \geq 2$. It follows from Proposition 7.5, Theorem 7.4 and Proposition 1.5.

8. Case $S O(n, n)$ and $S O(2 n, \mathbb{C}), n \geq 4$.

Let us assume that $G=S O(n, n)$ or $G=S O(2 n, \mathbb{C})$. In this case $\Sigma\left(\mathfrak{g}_{0}, \mathfrak{a}_{0}\right)$ is of type $D_{n}$ and, if as usual we identify $\mathfrak{a} \simeq \mathbb{C}^{n}$, then the restricted roots become $\left\{ \pm e_{i} \pm e_{j}\right\}$. Define

$$
r= \begin{cases}1, & \text { if } G=S O(n, n) \\ 2, & \text { if } G=S O(2 n, \mathbb{C}) .\end{cases}
$$


Here, $J(\nu)$ integral infinitesimal character if and only if

$$
\nu_{i} \in r \mathbb{Z}+r \frac{\epsilon}{2}, \text { with } \epsilon=0,1 .
$$

Again, we can assume that

$$
\nu_{1} \geq \nu_{2} \geq \cdots \geq\left|\nu_{n}\right| \geq 0
$$

and define

$$
R(i)=R_{\nu}(i)=\#\left\{j: \nu_{j}=r\left(i+\frac{\epsilon}{2}\right)\right\}, \quad i \geq 1
$$

and

$$
R(0)=R_{\nu}(0)=(2-\epsilon) \#\left\{j: \nu_{j}=r \frac{\epsilon}{2}\right\}+(1-\epsilon) .
$$

Take $\nu \in \mathfrak{a}_{\text {int }}^{*}$, then $J(\nu)$ admits an invariant Hermitian form if and only if $n$ is even, or $n$ is odd, $\epsilon=0$ and $R(0)>1$. Let us see the following:

Theorem 8.4. If $J(\nu)$ has integral infinitesimal character, then an invariant Hermitian form is positive definite on $J(\nu)^{\mu}, \mu \in \mathfrak{p}$, if and only if the following conditions are satisfied:

(1) $R(i+1) \leq R(i)+1$, for $i \geq 0$;

(2) $R(i+1)=R(i)+1$, for $i \geq 1$, then $R(i)$ is odd;

(3) $R(0)$ is odd;

(4) $R(0)>1$.

Proof. See Theorem 10.3 in $[\mathbf{B J}]$. The condition $R(0)>1$ does not appear in the statement of this theorem in $[\mathbf{B J}]$, but it is easy to see, checking the proof, that, otherwise, $q_{\mu}(\nu)>0$.

Now, we can prove the following:

Proposition 8.5. Consider $\nu \in \mathfrak{a}_{\mathrm{int}}^{*}$ such that $J(\nu)$ has integral infinitesimal character. If conditions (1)-(4) above are satisfied then $\nu=\sum_{\alpha \in \Sigma^{+}} c_{\alpha} \alpha$ with $c_{\alpha}=1 / 2$ or 0 .

Proof. Let us assume that $r=1, \epsilon=0$. Since the Weyl group is the group of permutation and sign changes involving an even number of signs of the set of $n$ elements, by condition (2) and $R(0)>1$, we can suppose that $\nu_{i} \geq 0$ and

$$
\nu=(k, \ldots, k, k-1, \ldots, k-1, \ldots \ldots, 1, \ldots, 1,0, \ldots, 0) .
$$

Since $R(0)>1$, we have $k-i \leq n-\sum_{j=0}^{i} R(k-j)$, and thus

$$
\nu=\frac{1}{2} \sum_{m=0}^{k-1} \sum_{r=1}^{R(k-m)} \sum_{t=1}^{k-m}\left[\left(e_{\mathcal{T}(m)+r}-e_{\mathcal{T}(m)+r+t}\right)+\left(e_{\mathcal{T}(m)+r}+e_{\mathcal{T}(m)+r+t}\right)\right],
$$


where $\mathcal{T}(m)$ was defined in (3.6). Now, let us assume that $\epsilon=1$ and $n$ even. So, by a conjugation by the Weyl group we can assume that $\nu_{i} \geq 0$ for $i=0, \ldots, n-1$ and moreover

$$
\begin{aligned}
\nu=\left(k+\frac{1}{2}, \ldots, k+\frac{1}{2}, k-1+\frac{1}{2}, \ldots, k-1+\frac{1}{2},\right. \\
\left.\ldots, \ldots, 1+\frac{1}{2}, \ldots, 1+\frac{1}{2}, \frac{1}{2}, \ldots, \pm \frac{1}{2}\right) .
\end{aligned}
$$

Since $R(0)>1$ and by condition (3) in Theorem 8.4 we can prove that $k-i+1 \leq n-\sum_{j=0}^{i} R(k-j)$, and defining $\delta=\left\{\begin{array}{ll}1, & \text { if } \nu_{n}=\frac{1}{2} \\ 0, & \text { if } \nu_{n}=-\frac{1}{2} .\end{array}\right.$ we can rewrite $\nu$ as follows

$$
\begin{aligned}
\nu=\sum_{m=0}^{k-1} & \sum_{r=1}^{R(k-m)} \sum_{t=1}^{k-m} \frac{1}{2}\left\{\left[\left(e_{\mathcal{T}(m)+r}-e_{\mathcal{T}(m)+r+t}\right)+\left(e_{\mathcal{T}(m)+r}+e_{\mathcal{T}(m)+r+t}\right)\right]\right. \\
& \left.+\left(e_{\mathcal{T}(m)+r}+(-1)^{\mathcal{T}(m)+r+\delta} e_{n}\right)\right\}+\sum_{j=1}^{R(0)-1} \frac{1}{2}\left(e_{\mathcal{T}(k)+j}+(-1)^{j} e_{n}\right)
\end{aligned}
$$

where $\mathcal{T}(m)$ was defined in (3.6). And, since $n$ is even and $R(0)$ is odd, we have completed our proof. The case $S O(2 n, \mathbb{C})$ follows as above using multiplicities of positive roots for this particular case.

Then we can give the:

Proof of Theorem 2.1 for $S O(n, n)$ and $S O(2 n, \mathbb{C}), n \geq 4$. It is immediate from Proposition 8.5, Theorem 8.4 and Proposition 1.5.

\section{References}

[BJ] J. Bang-Jensen, On unitarity of spherical representations, Duke Math J., 61 (1990), 157-194.

[BJ2] - The multiplicities of certain K-types in spherical representations, J. Funct. Anal., 91 (1990), 346-403.

[HJ] S. Helgason and K. Johnson, The bounded spherical functions on symmetric spaces, Advances in Math., 3 (1969), 587-593.

[Kn] A. Knapp, Lie Groups Beyond an Introduction, Progress in Mathematics, Birkhauser, Vol. 140, 1996.

[K] B. Kostant, On the Existence and Irreducibilitiy of Certain Series Representations, Proceedings of the summer school on Groups representations, Bolyai Jonos Math. Society, Budapest, 1971.

[SV] S. Salamanca-Riba and D. Vogan, On the classification of unitary representation of reductive groups, preprint. 
[V] D. Vogan, Representations of Real Reductive Lie Groups, Progress in Mathematics, Birkhauser, Vol. 15, 1981.

[V2] Unitarizability of certain series of representations, Annals of Math., 119 (1984), 141-187.

Received May 5, 1999 and revised December 9, 1999. This research was partially supported by Fundación Antorchas and CONICET.

FAmaf - Universidad Nacional de Córdoba

Ciudad Universitaria, Córdoba (5000)

Argentina

E-mail address: boyallia@mate.uncor.edu 